\title{
Interaction study of three overlapping synthetic peptides belonging to E2 protein of GBV-C/HGV with liposomes as biomembrane models
}

\author{
N. Rojo ${ }^{1}$, M. Muñoz ${ }^{2}$, M. Pujol ${ }^{2}$, M.A. Alsina ${ }^{2}$ and I. Haro ${ }^{1}$ \\ 1 Department of Peptide \& Protein Chemistry. Institute for Chemical and Environmental \\ Research (IIQAB-CSIC), Jordi Girona 18-26, 08034 Barcelona, Spain \\ ${ }^{2}$ Unidad Asociada CSIC: "Péptidos y Proteínas: Estudios fisicoquímicos", Department of \\ Physicochemistry, Faculty of Pharmacy, University of Barcelona, Av. Joan XXIII s/n, \\ 08028 Barcelona, Spain
}

\begin{abstract}
In this work we studied by DSC the interaction of three antigenic overlapping peptides belonging to the E2 envelope protein of Hepatitis G virus, namely E2(39-53), E2(32-53) and E2(26-53), with liposomes of different lipid composition (DPPC, DMPC and DMPG) as biomembrane models. The effect of the three selected peptides on the thermotropic behaviour of lipid bilayers has been evaluated.
\end{abstract}

\section{INTRODUCTION}

The Hepatitis G virus (GBV-C/HGV) was first identified in 1994 from the plasma of a patient with etiologically unexplained chronic hepatitis. The Hepatitis G virus belongs to the family of Flaviviridae [1].

GBV-C/HGV infections were detected in approximately 1-2\% of the general population and in up to $30 \%$ of risk groups (e.g. drug addicts). GBV-C/HGV can be transmitted parenterally, sexually and from GBV-C/HGV infected mothers to childs. The majority of GBV-C/HGV-infected individuals have no clinical or biochemical evidence of liver disease. However, there are cases of acute, including fulminated hepatitis and chronically hepatitis, with their sequelae where GBV-C/HGV is the only recognizable cause of liver disease [2]. The 5'-end of the genome codes for two structural proteins: E1 and E2, the non-structural proteins NS2,NS3,NS4, NS5a and NS5b being located at the 3 '-terminal region.

The distribution of protein or peptide drugs within a lipid carrier is important, since interactions between the lipid and the drug not only affect the physicochemical properties of the protein/peptide but also its release rate. Differential scanning calorimetry (DSC) is a powerful tool for studying protein/peptide-lipid interactions following the thermotropic behaviour of phospholipids in the presence of the protein or the peptide [3]. 


\section{EXPERIMENTAL PART}

\subsection{Peptide synthesis}

Experimental details of the synthetic procedures will be described elsewhere. To summarise the procedures briefly: E2(39-53): GNVTLLCDCPNGPWV; E2(32-53):GERVWDRGN VTLLCDCPNGPWV and E2(26-53):GSRVPTG ERVWDRGNVTLLCDCPNGPWV peptide sequences were obtained manually on a Rink amide MBHA $(0.75 \mathrm{meq} / \mathrm{g})$ resin by solid phase methodology following a 9-Fluorenylmethoxycarbonyl (Fmoc)/ tert-butyl (tBut) strategy by means of a N, N' -diisopropylcarbodiimide (DIPCD)/1-hidroxybenzotriazole (HOBt) activation.

Threefold molar excesses of Fmoc-amino acids were used throughout the synthesis. The stepwise addition of each residue was assessed by the Kaiser's (ninhydrin) test. Desprotection was performed in $20 \%$ piperidine/DMF.

Different conditions were tested to cleave the peptide from the resin and to remove the side-chain blocking groups. Overall, peptide resins were treated with TFA solutions containing appropriate scavengers $\left(5 \% \mathrm{H}_{2} \mathrm{O}, 3 \%\right.$ ethanedithiol, $5 \%$ anisol).

Crude peptides were purified by preparative high performance liquid chromatography (HPLC) on a Shimadzu chromatography equipped with a $\mathrm{C}_{18}$-silica column. Purified peptides were characterized by analytical HPLC, amino acid analysis and electrospray mass spectrometry [4].

\subsection{Differential scanning calorimetry}

DSC experiments were carried out with multilamellar vesicles (MLVs) composed of DPPC, DMPC and DMPG and were performed using a DSC 821E Mettler Toledo calorimeter (Greifensee, Switzerland). Hermetically sealed aluminium reference and sample containing pans were used. Sample pans were loaded by adding $30 \mu \mathrm{l}$ of vesicle suspension, corresponding to approximately 0.13 $\mathrm{mg}$ of DPPC, $0.15 \mathrm{mg}$ of DMPC and $0.31 \mathrm{mg}$ of DMPG. Differences in the heat capacity between the sample and the reference cell were obtained by raising the temperature at a constant rate of $5^{\circ} \mathrm{C} / \mathrm{min}$ over the range from $0^{\circ} \mathrm{C}$ to $60^{\circ} \mathrm{C}$ (DPPC vesicles) and from $0^{\circ} \mathrm{C}$ to $40^{\circ} \mathrm{C}$ (DMPC and DMPG vesicles). All samples were submitted to three heating/cooling cycles. Data from the first scan were always discarded to avoid false results coming to the possible lipid-peptide mixing in the sample under heating. The endothermic peak coming from the second scan of the control sample was used as a reference template. The instrument was calibrated with Indium. To ensure scan-to-scan reproducibility three consecutive scans of the same sample were performed. DSC runs were carried out within the same day of liposomes preparation. Molar enthalpies of transition $(\Delta \mathrm{H})$ were calculated from peak areas by means of a START ${ }^{\mathrm{e}}$ Mettler Toledo system software.

\section{RESULTS AND DISCUSSION}

The thermotropic phase behaviour of zwitterionic DPPC vesicles was studied by DSC, in the absence and in the presence of increasing concentrations of GBV-C/HGV peptides. At high lipid-to peptide ratios, the chain melting transition of DPPC was not affected by the three overlapping synthetic peptides (Figure 1).

The interaction of small amounts of the E2 synthetic peptides reported in this paper do not alter significantly the packing of hydrocarbon chains in the gel and liquid-crystalline states in DPPC MLVs. 


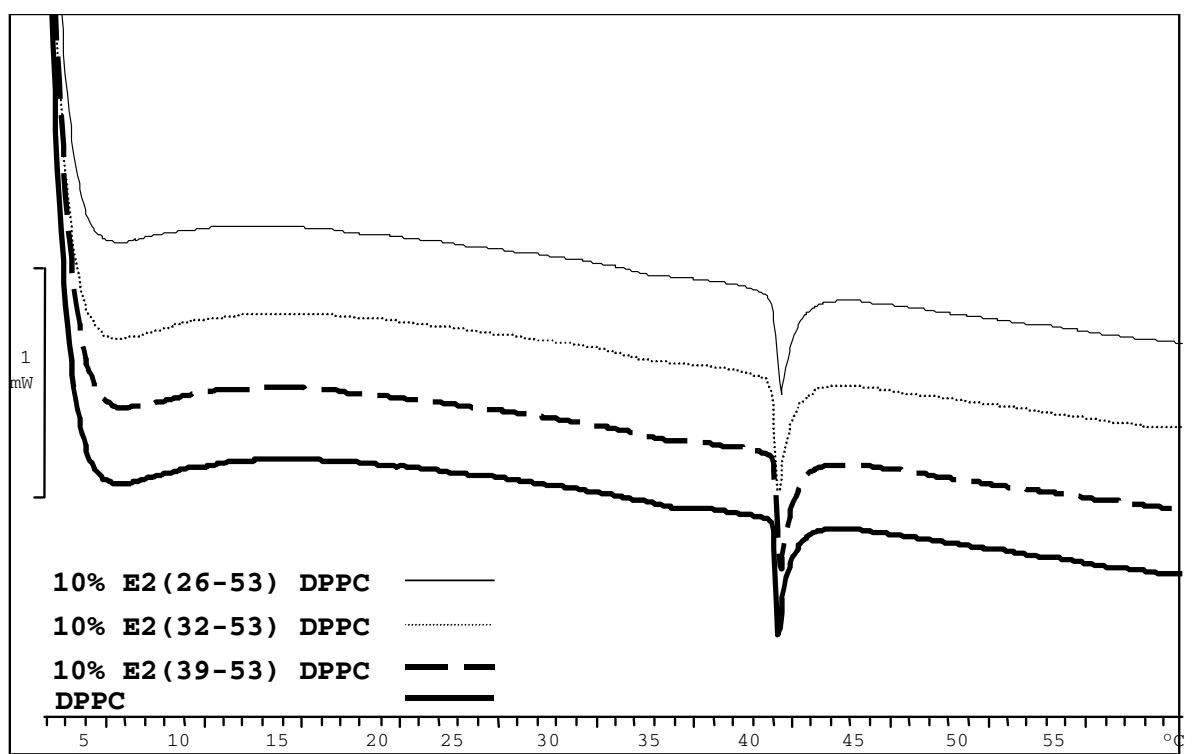

Figure 1. DSC thermograms of DPPC bilayers in the absence and in the presence of $10 \%$ of E2(26-53), E2(32-53) and E2(39-53) peptide sequences. The peptides concentration is given in mol\% with respect to lipid.

On the other hand, as previously obtained for other synthetic peptides [5] the addition of $10 \%$ of the E2 peptides clearly diminished the enthalpy of the main transition peak in DMPC vesicles (Figure 2).

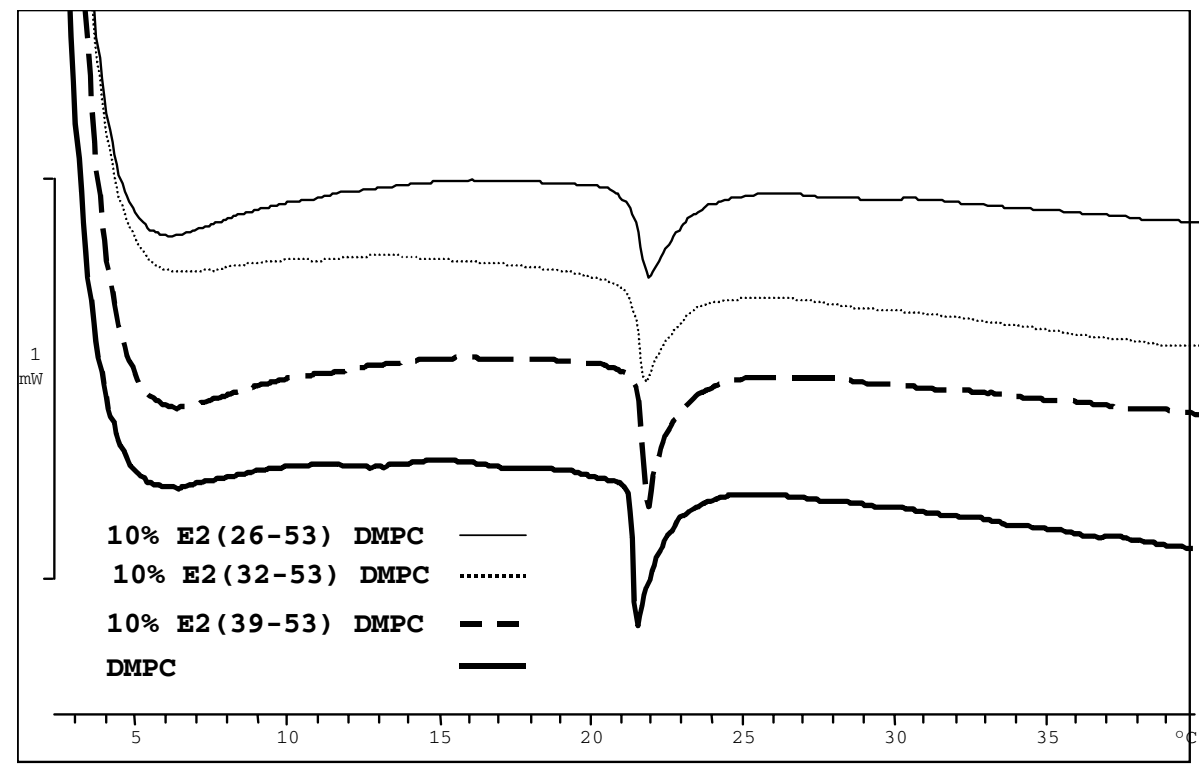

Figure 2. DSC thermograms of DMPC bilayers in the absence and in the presence of $10 \%$ of E2(26-53), E2(32-53) and E2(39-53) peptide sequences. The peptides concentration is given in mol\% with respect to lipid.

The effect of E2 peptides on gel/liquid-crystalline transition enthalpy $(\Delta \mathrm{H})$, and the temperature width at half-height of the heat absorption peak $\left(\Delta \mathrm{T}_{1 / 2}\right)$ for pure DMPG vesicles and at different percentages of E2-peptides is shown in Table 1. 
For the mixtures tested, we can observe that the enthalpy diminished and $\Delta \mathrm{T}_{1 / 2}$ were much higher, indicating the incorporation of the peptide into the lipid bilayer and the subsequent binding and penetration of the peptide into the hydrophobic core of the bilayer.

Table 1. Gel/Liquid-Crystalline Transition enthalpies, $\Delta \mathrm{H}$, and the temperature width at half-height of the heat absorption peak $\left(\Delta \mathrm{T}_{1 / 2}\right)$ of E2-peptides as obtained from DSC.

\begin{tabular}{|c|c|c|}
\hline & $\Delta \mathrm{H}(\mathrm{KJ} / \mathrm{mol})$ & $\Delta \mathrm{T}_{1 / 2}\left({ }^{\circ} \mathrm{C}\right)$ \\
\hline$D M P G$ & 25.7 & 1.4 \\
\hline \multicolumn{3}{|c|}{ DMPG/E2(39-53) } \\
\hline 99/1 & 26.3 & 1.4 \\
\hline $95 / 5$ & 27.1 & 1.4 \\
\hline $90 / 10$ & 22.2 & 1.5 \\
\hline $80 / 20$ & 21.1 & 1.6 \\
\hline \multicolumn{3}{|c|}{ DMPG/E2(32-53) } \\
\hline 99/1 & 29.1 & 1.3 \\
\hline $95 / 5$ & 26.6 & 2.9 \\
\hline $90 / 10$ & 20.5 & 3.1 \\
\hline $80 / 20$ & - & - \\
\hline \multicolumn{3}{|c|}{ DMPG/E2(26-53) } \\
\hline 99/1 & 26.5 & 1.8 \\
\hline $95 / 5$ & 22.1 & 5.2 \\
\hline $90 / 10$ & 15.1 & 7.1 \\
\hline $80 / 20$ & & \\
\hline
\end{tabular}

These data indicate that the peptides disrupt the packing of the DMPG bilayer, as they interdigitate and cause perturbations that contribute to the bilayer fluidity. These effects were slightly more pronounced for E2(26-53) than for E2(32-53) and E2(39-52) peptides (Figure 3, Table 1).

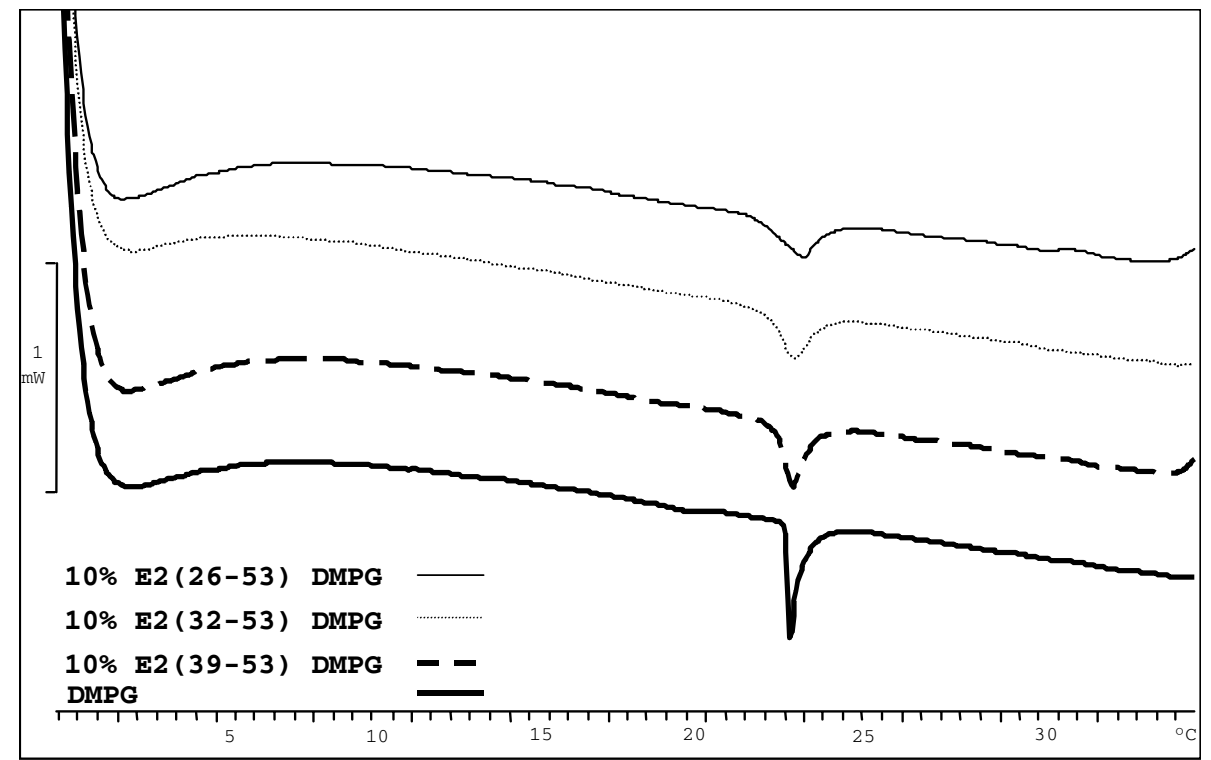

Figure 3. DSC thermograms of DMPG bilayers in the absence and in the presence of $10 \%$ of E2(26-53), E2(32-53) and E2(39-53) peptide sequences. The peptides concentration is given in mol\% with respect to lipid. 
This interaction is probably more favourable between the anionic lipid (DMPG) and the peptide E2(26-53) that is zwiterionic at $\mathrm{pH} \mathrm{7.4,} \mathrm{than} \mathrm{the} \mathrm{established} \mathrm{between} \mathrm{DMPG} \mathrm{with} \mathrm{the} \mathrm{anionic}$ peptides, this is E2(39-53) and E2(32-53). Therefore, electrostatic forces seem to play an important role in the observed by DSC interactions between the synthetic E2 peptides and the studied phospholipids.In summary, the synthetic GBV-C/HGV E2 peptides incorporate into DMPG lipid bilayers perturbing the packing of lipids and affecting their thermotropic properties. From our results and according Papahadjopoulos et al [6], it is tentatively suggested that the studied peptides preferentially locate at the outer regions of the bilayer extending the interactions to the polar headgroups followed by a partial penetration and deformation of the bilayer. In conclusion, this study has revealed that the extension of the perturbation effect of the peptides was related with the chain length as well as with the net charge of E2 HGV/GBV-C peptides.

\section{Acknowledgements}

This work was funded by Grants BQU2003-05070-CO2-01/02.

\section{References}

[1] Kiyosawa K, Tanaka E, Intervirology, 42, (1999), 185-195

[2] Tacke M, Kiyosawa K, Stark K, Schlueter V, Ofenloch-Haehnle B, Hess G, Engel A. The Lancet, 349, (1997), 318-320

[3] Lohner K, Prenner EJ., Biochim Biophys Acta, 1462, (1999), 141-156

[4] Rojo N, Ercilla G, Haro I., Current Protein Peptide Science, 4, (2003), 291-298

[5] Rojo N, Gómara M J, Busquets M A, Alsina M A, Haro I., Talanta, 60, (2003), 395-404

[6] D. Papahadjopoulos, M. Moscarello, E.H. Eylar, T. Isac, BBA 401, (1975), 317-335 